\title{
Perceiving vowels from uniform spectra: Phonetic exploration of an auditory aftereffect
}

\author{
QUENTIN SUMMERFIELD, MARK HAGGARD, JOHN FOSTER, \\ and STUART GRAY \\ MRC Institute of Hearing Research, University of Nottingham, Nottingham, England
}

\begin{abstract}
A carefully spoken vowel can generally be identified from the pattern of peaks and valleys in the envelope of its short-term power spectrum, and such patterning is usually necessary for the identification of the vowel. The present experiments demonstrate that segments of sound with uniform spectra, devoid of peaks and valleys, can be identified reliably as vowels under certain circumstances. In Experiment 1, 1,000 msec of a segment whose spectrum contained peaks in place of valleys and vice versa (i.e., the complement of a vowel) preceded a 25 -msec spectral amplitude transition, during which the valleys became filled, leading into a 250 -msec segment with a uniform spectrum. The segment with the uniform spectrum was identified as the vowel whose complement had preceded it. Experiment 2 showed that this effect was eliminated if the duration of the complement was less than $150 \mathrm{msec}$, if more than $500 \mathrm{msec}$ of silence separated the uniform spectrum from the complement, or if the uniform spectrum and the complement were presented to different ears. This third result and comparisons with parameters of auditory aftereffects obtained by others with nonspeech stimuli suggest that the effect is rooted in peripheral adaptation processes and that central processes responsible for selective attention and perceptual grouping play only a minor role at most. Experiment 3 demonstrated that valleys in the spectral structure of a complement need be only $2 \mathrm{~dB}$ deep to generate the effect. The effect should therefore serve to enhance changes in spectral structure in natural speech and to alleviate the consequences of uneven frequency responses in communication channels.
\end{abstract}

In a pilot experiment, we established that waveforms with uniform spectra, devoid of peaks and valleys, can be identified reliably as different vowels under certain circumstances. We synthesized waveforms whose spectra were complementary to those of rectangular approximations to the vowels $/ i /$ and $/ a /$. These complementary spectra had peaks in place of valleys and vice versa. We created stimuli in which a 400-msec segment of waveform with a uniform flat spectrum was surrounded by two 500 -msec segments of one of the two vowel complements. Fifty-millisecond spectral amplitude transitions linked the two types of waveform. The segments with uniform spectra sounded like the vowels whose complements surrounded them. Taking a more realistic vowel spectrum as a starting point, Figure 1 displays a progression of power spectra computed at 50 -msec intervals during a sequence in which the complement of the vowel /a/ surrounds a segment with a uniform spec-

The basic phenomenon discussed in this paper was demonstrated by Stuart Gray before his tragic death in a road accident in December 1977. Some of the data reported here were presented to the 101st Meeting of the Acoustical Society of America, Ottawa, Ontario, May 1981, and to the summer meeting of the Experimental Psychology Society, Oxford, July 1981. We thank Carol Jameson for assistance in scoring the data and Andrew Sidwell, Matthew McGrath, Christopher Darwin, and two reviewers for their comments on earlier versions of the paper. Quentin Summerfield's mailing address is: MRC Institute of Hearing Research, University Park, Nottingham, NG7 2RD, England. trum (lower panel) and during a sequence in which the vowel $/ a /$ itself alternates with silence (upper panel). The middle segment of each stimulus sounds like "AH."

At least three factors, discussed in detail below, could contribute to an effect of this type: (1) Listeners may use the spectral distribution of the first temporal derivative of local spectral amplitude directly as a cue to the identity of an isolated vowelthe "spectral change" hypothesis. (2) Listeners may use common covariation in amplitude to group perceptually a set of harmonics, thereby separating them from unrelated changes in spectral amplitude in background noises-the "perceptual grouping" hypothesis. (3) Adaptation may occur during the presentation of the first segment of the complement, resulting in an enhanced auditory representation of spectral energy at the onset of uniform spectrum at frequencies corresponding to the valleys in the complement-the "adaptation" hypothesis.

These hypotheses are interpretative and relate to different levels of auditory and cognitive processing. Thus, they need not be mutually exclusive, and the one to be emphasized may depend upon particular factors in the task under consideration and the purpose of the explanation adopted.

\section{Spectral Change Hypothesis}

Plots of rate of change of spectral amplitude by 


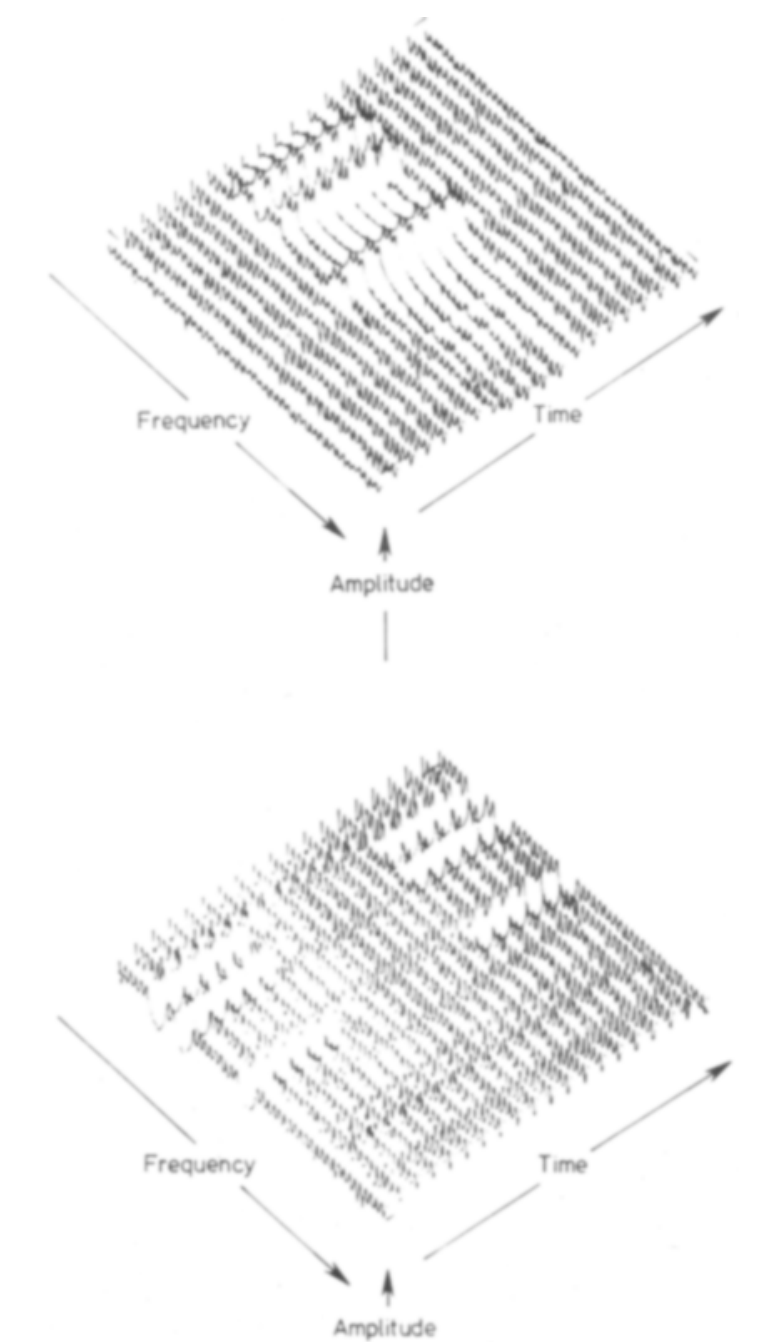

Figure 1. Progressions of power spectra computed at 50-msec Intervals during a sequeace in which $\mathbf{4 0 0}$ msec of a synthetic threeformant approxdmution to / $/$ / are surrounded by sllence (upper panel) and in which a 400-muec segment with a uniform spectrum is sarrounded by two 500 -meec segments whowe spectra are the complement of $/ \mathbf{a}$ / (lower panel). In the spectrum of the vowel complement, formant peaks are replaced by valleys and vice versa. In these sequences, both the aynthetic vowel and the segment with the uniform spectram are identified as "ah."

time and frequency would look the same over the central 1,000 msec of the stimuli displayed in Figure 1 . The function would be nonzero during the spectral amplitude transitions at frequencies corresponding to the peaks in the vowel spectrum, and zero at other times and frequencies. Possibly this information can contribute to the identity of an isolated vowel. Its potential importance is suggested by the subjective observation that if one synthesizes a vowel waveform and plays it for longer than a few seconds, it sounds most vowel-like at its onset; it may then loose its identity, but partially regain it when gated off. Changes in fundamental frequency and in formant frequency have similar refreshing effects. In other words, the vowel appears to be specified most clearly at times when spectral amplitude transitions are occurring, which serve to clarify its spectrum, probably most potently around the frequencies corresponding to its formants. It would be difficult to dissociate the "spectral change" hypothesis from the "adaptation" hypothesis when considering positive-going spectral amplitude transitions. However, the hypothesis that negative-going spectral amplitude transitions at the offset of an isolated vowel can serve to specify or enhance its identity can be tested by presenting a waveform with a uniform spectrum followed by spectral amplitude transitions leading into vowel complements. Sensitivity to negativegoing spectral amplitude transitions could allow listeners to identify the offsets of segments with uniform spectra as the vowels whose complements followed them.

\section{Perceptual Grouping Hypothesis}

An alternative hypothesis is that listeners use common variation in amplitude in different spectral regions as a basis for grouping the components of a sound so as to distinguish it from background noises. Traditionally (Koffka, 1922) and more recently (e.g., Dannenbring \& Bregman, 1978; Darwin, 1983; Hall, Haggard, \& Fernandes, in press; Marr, 1982), it has been argued that processes that select structure from noise must operate either prior to, or in concert with, processes that detect those attributes of structured signals which determine their identity. Common variation in amplitude may help to group formants in audition.

\section{Adaptation Hypothesis}

A third possibility is that a form of adaptation occurs during the presentation of the initial segment of the complement which results in an enhancement of the auditory response to energy at frequencies corresponding to the formants of the vowel when the segment with the uniform spectrum is presented. It has long been known that exposure to a notched noise can induce a tonal aftereffect (Zwicker, 1964). Two other results, also obtained with nonspeech stimuli, are more directly analogous to the present phenomenon and suggest that some form of adaptation may play a role in each of them. The first was discovered independently by several authors, whose results were summarized by Viemeister (1980). Consider a complex tone composed of sine waves whose frequencies are integer multiples of $200 \mathrm{~Hz}$. The $1000-\mathrm{Hz}$ component is then omitted. If the incomplete harmonic series is presented for 1 to $2 \mathrm{sec}$ and then followed by the complete series, the $1000-\mathrm{Hz}$ component stands out from the other harmonics and can be heard distinctly. This result can be seen as a 
limiting instance of the present effect. In another parallel, Wilson (1970) synthesized a noise whose spectrum was sinusoidally rippled. If this sound was followed by a noise with a flat, uniform spectrum, the uniform noise was heard as having a spectral ripple complementary to that in the first segment and hence as having a pitch. Parameters of the effect could be measured by determining the depth, and the rate of decay, of spectral amplitude modulation required to cancel the aftereffect. Viemeister and Bacon (1981) suggested that these effects demonstrated not so much the adaptation of the auditory response to frequency-specific stimulation as the adaptation of suppression processes. In their critical experiment, they compared the amount of forward masking produced by a $2000-\mathrm{Hz}$ tone, first, when it was a member of a harmonic series with a fundamental of $200 \mathrm{~Hz}$ and, second, when it was introduced into a harmonic series from which it had been missing. On average, an additional $8 \mathrm{~dB}$ of masking was produced in the second condition, suggesting that the aftereffect depends primarily on previously missing components achieving an enhanced auditory representation, rather than on previously present components declining to a diminished representation.

The experiments reported here are located more in the ecological than in the physiological realm, and do not explore the issue of the adaptation of suppression directly. Rather, they were designed to establish the extent and chief parameters of the effect found in the pilot study. They show first that the phenomenon can be used to generate percepts of vowels, and so the accuracy of vowel identification can be used as a dependent measure with which to explore parameters of the effect. We shall show that approximately $150 \mathrm{msec}$ of the complement of a vowel is required to establish the effect, that it is eliminated when between 100 and $500 \mathrm{msec}$ of silence (depending on the listener) intervene between complement and uniform spectrum, and that the effect is not obtained if vowel complements and uniform spectra are presented to different ears. It is concluded that the phenomenon does play a useful role in the natural perception of speech.

\section{GENERAL METHOD}

All stimuli were created digitally $(10,000$ samples/sec, 12-bit amplitude resolution). Initially, a waveform with a uniform spectrum was synthesized by summing the first 50 harmonics of $100 \mathrm{~Hz}$ in random phase. The amplitudes of the harmonics were attenuated by $6 \mathrm{~dB}$ per octave above $100 \mathrm{~Hz}$ to approximate the longterm spectrum of natural periodically excited speech Panel a of Figure 2 displays the power spectrum of such a segment. From it, three other types of segment, vowels, complements, and indistinct vowels, were derived.

Five vowel segments were defined by eliminating all but nine harmonics arrayed in three groups of three adjacent harmonics, with the middle members of each group centered approximately on the center frequencies of the first three formants of the five distinctive vowels $/ \mathrm{i} /, / \mathrm{a} /, / \mathrm{u} /, / 2 /, / \mathcal{J} /$. The first four occur
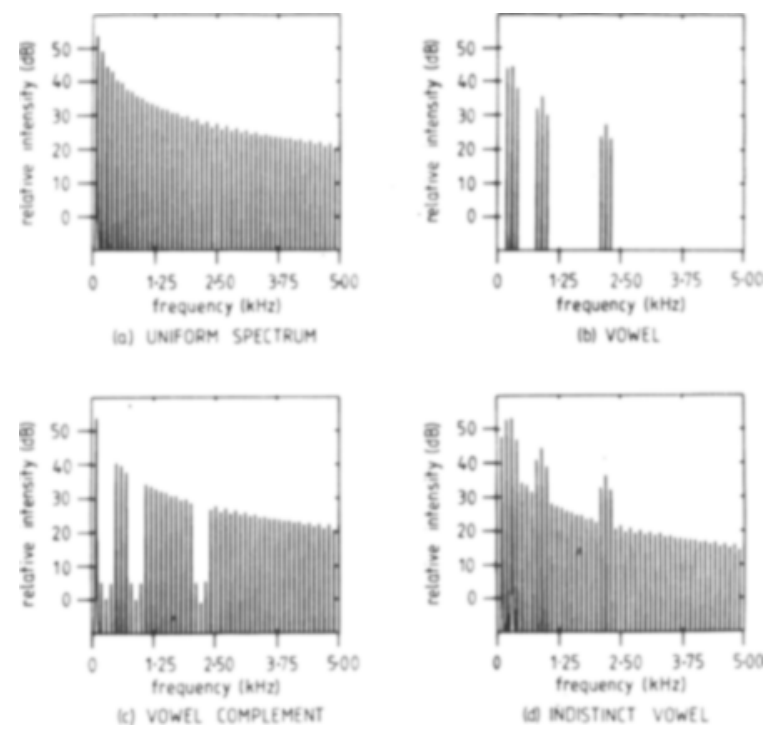

Figure 2. Power spectra of examples of the four basic types of segment incorporated in the stimuli of Experiment 1. (a) The spectrum of a segment with a uniform spectrum. The amplitudes of the 50 harmonles of $100 \mathrm{~Hz}$ decrease at $6 \mathrm{~dB}$ per octave above $100 \mathrm{~Hz}$. Apparent ripple results from the difference between the frequency sampling of the spectral analysis and the frequency spacing of the harmonics. (b) The spectrum of the vowel $/ u /$. Three groups of three harmonics approximately centered on the frequencies of the first three formants of $/ u$ / have been retained from the segment with the uniform spectrum, and the amplitudes of the outlying members of each trio have been reduced by $5 \mathrm{~dB}$. (c) The spectral complement of $/ u /$. Compared with the segment with the uniform spectrum, the amplitudes of nine harmonics have been reduced by their amplitudes in the vowel $/ \mathbf{a} /$. (d) The spectrum of a segment of the indistinct vowel $/ u /$. Compared with the uniform spectrum, the amplitudes of the three groups of three harmonics comprising the vowel $/ u$ / have been raised by $10 \mathrm{~dB}$ (outlying members of each trio) and by $15 \mathrm{~dB}$ (central members of each trio).

with very similar formant frequencies in British and American English. The form of $/ 3 /$ used occurs only in American English. Relative to their levels in the uniform spectrum, the levels of the outlying members of each trio were attenuated by $5 \mathrm{~dB}$ to shape these pseudoformants. Panel $b$ of Figure 2 displays the power spectrum of the vowel $/ u /$. Table 1 lists the frequencies of the harmonics used to synthesize each of the five vowels. Intensities have been specified relative to a uniform spectrum, rolling off at $6 \mathrm{~dB}$ per octave, with the level of the $100-\mathrm{Hz}$ harmonic at $55 \mathrm{~dB}$.

Five vowel-complement segments were defined by eliminating from the uniform spectrum the nine harmonics that would be present in the corresponding vowel. Panel $c$ of Figure 2 displays the power spectrum of the complement of the vowel $/ u /$.

Finally, five indistinct vowel segments were derived from the uniform spectrum by raising the levels of the nine harmonics comprising each vowel by $10 \mathrm{~dB}$ (for the outlying members of each trio) and by $15 \mathrm{~dB}$ (for the central member of each trio). Panel d of Figure 2 displays the power spectrum of the indistinct vowel $/ \mathbf{u} /$.

The stimuli used in the experiments reported below were derived from these four basic types of segment. Stimuli were output at 10,000 samples/sec through 12-bit digital-to-analog converters (DEC PDP-11/60, LPA11K), low-pass filtered at $4250 \mathrm{~Hz}$ (KEMO VBF/8, $-96 \mathrm{~dB} /$ octave), and recorded on audio tape. In listening tests, the tapes were played to listeners binaurally, except in Experiment $2 c$, through TDH-39 headphones with the level of the 100-Hz harmonic at $74 \mathrm{~dB}$ SPL (B\&K Type 4253 wide-band artificial ear, Type 4134 microphone, and Type 2203 sound-level 
Table 1

Relative Intensities (dB) of the Harmonics (Hz) of $100 \mathrm{~Hz}$ Defining the Five Vowel Stimuli

\begin{tabular}{|c|c|c|c|c|c|c|c|c|c|}
\hline \multicolumn{10}{|c|}{ Vowels } \\
\hline \multicolumn{2}{|c|}{ EE } & \multicolumn{2}{|c|}{$\mathrm{AH}$} & \multicolumn{2}{|c|}{$\mathrm{OO}$} & \multicolumn{2}{|c|}{ OR } & \multicolumn{2}{|c|}{ ER } \\
\hline $\mathrm{Hz}$ & $\mathrm{dB}$ & $\mathrm{Hz}$ & $\mathrm{dB}$ & $\mathrm{Hz}$ & $\mathrm{dB}$ & $\mathrm{Hz}$ & $\mathrm{dB}$ & $\mathrm{Hz}$ & $\mathrm{dB}$ \\
\hline \multicolumn{10}{|c|}{ Formant: F1 } \\
\hline 200 & 44.00 & 600 & 34.49 & 200 & 44.00 & 400 & 38.00 & 400 & 38.00 \\
\hline 300 & 45.49 & 700 & 38.16 & 300 & 45.49 & 500 & 41.07 & 500 & 41.07 \\
\hline 400 & 38.00 & 800 & 32.00 & 400 & 38.00 & 600 & 34.49 & 600 & 34.49 \\
\hline \multicolumn{10}{|c|}{ Formant: F2 } \\
\hline 2200 & 23.24 & 1000 & 30.07 & 800 & 32.00 & 700 & 33.16 & 1300 & 27.80 \\
\hline 2300 & 27.86 & 1100 & 34.24 & 900 & 35.98 & 800 & 37.00 & 1400 & 32.16 \\
\hline 2400 & 22.49 & 1200 & 28.49 & 1000 & 30.07 & 900 & 30.98 & 1500 & 26.56 \\
\hline \multicolumn{10}{|c|}{ Formant: F3 } \\
\hline 2900 & 20.85 & 2300 & 22.86 & 2100 & 23.65 & 2300 & 22.86 & 1600 & 26.00 \\
\hline 3000 & 25.56 & 2400 & 27.49 & 2200 & 28.24 & 2400 & 27.49 & 1700 & 30.48 \\
\hline 3100 & 20.27 & 2500 & 22.14 & 2300 & 22.86 & 2500 & 22.14 & 1800 & 24.98 \\
\hline
\end{tabular}

meter, linear weighting) (55 dBA). The level of the segment with the uniform spectrum was $78 \mathrm{~dB}$ SPL (Linear) (71 dBA).

\section{Subjects}

A screening test was prepared by recording a randomized sequence of test stimuli derived from the five vowel segments. The duration of each stimulus was $300 \mathrm{msec}$, with rise and fall times of $25 \mathrm{msec}$ (linear in voltage and time). The stimuli were presented at the rate of one every $4 \mathrm{sec}$. Eight subjects, graduate students and members of staff of the Institute of Hearing Research, listened to the sequence. They were instructed to identify each stimulus as one of the sounds "ee," "ah," "oo," "or," and "er" by marking a response sheet. (In Southern British English, orthographic "or" is pronounced as a pure vowel with no $/ r /$. All of the listeners were familiar with the American English $/ 3 /$.) Six of the eight subjects scored over $95 \%$ correct. The other two scored closer to $80 \%$. In the three experiments reported below, data were gathered only from the former six. They ranged in age from 23 to 31 years and had audiometrically normal hearing. They included two of the authors.

\section{EXPERIMENT 1}

Experiment 1 was intended to establish first that stimuli akin to those displayed in the lower panel of Figure 1 can generate percepts of a sufficient range of vowel sounds to allow accuracy of identification to be used as the dependent measure in explorations of other parameters of the effect. The second goal was to determine whether perceptual sensitivity to spectralamplitude changes, in the sense proposed by the spectral change hypothesis, operated symmetrically. Two types of test stimulus were created. In the first, the complement of one of the five different vowels preceded a spectral amplitude transition leading into a uniform spectrum. In the second, the order of the segments was reversed; the uniform spectrum preceded a spectral amplitude transition leading into a vowel complement. If perceptual sensitivity to increases of spectral amplitude per se is the basis of the effect, listeners would identify the uniform spectrum as a vowel only if the complement preceded it. How- ever, if a full representation of physical changes in derivative terms is extracted, the offset of the segment with the uniform spectrum may sound like the vowel whose complement follows it.

One basic problem had to be overcome in this and subsequent experiments. The complements of vowels have a spectrum structure which, though unnatural, can be fairly vowel-like in some cases and can be categorized. For example, the complement of $/ \mathrm{i} /$ sounds a little like /a/, and vice versa. Potentially, listeners might learn the correspondence between the sounds of the complements and the effects they sometimes produce when coupled with uniform spectra, and so bias their responses. To overcome this problem, on half the trials in this and subsequent experiments, "foil" stimuli were presented. For Experiment 1, two foils were created from each test stimulus by replacing the uniform spectrum by an indistinct vowel. The indistinct versions of the vowels were used, rather than the vowels themselves, to avoid creating a class of "clear" stimuli in opposition to an unclear class. In no case was a complement paired with its own indistinct vowel. This procedure uncouples the association of particular complements with particular identification responses. It allows the accuracy with which foils are identified to be a criterion of the reliability of a listener's responses provided the perceived identity of the foils is sufficiently robust not to be changed by the preceding complement.

\section{Method}

Two types of test stimulus were synthesized along with appropriate foils. In the complement-to-uniform (CU) stimuli, $1,000 \mathrm{msec}$ of the complement of a vowel was followed by a 25 msec linear spectral amplitude transition leading into a 275-msec segment with a uniform spectrum, as schematized in Figure 3. During the spectral amplitude transition, the intensities of the 50 harmonics changed linearly in voltage and time from their values in the complement to their values in the uniform spectrum. The 


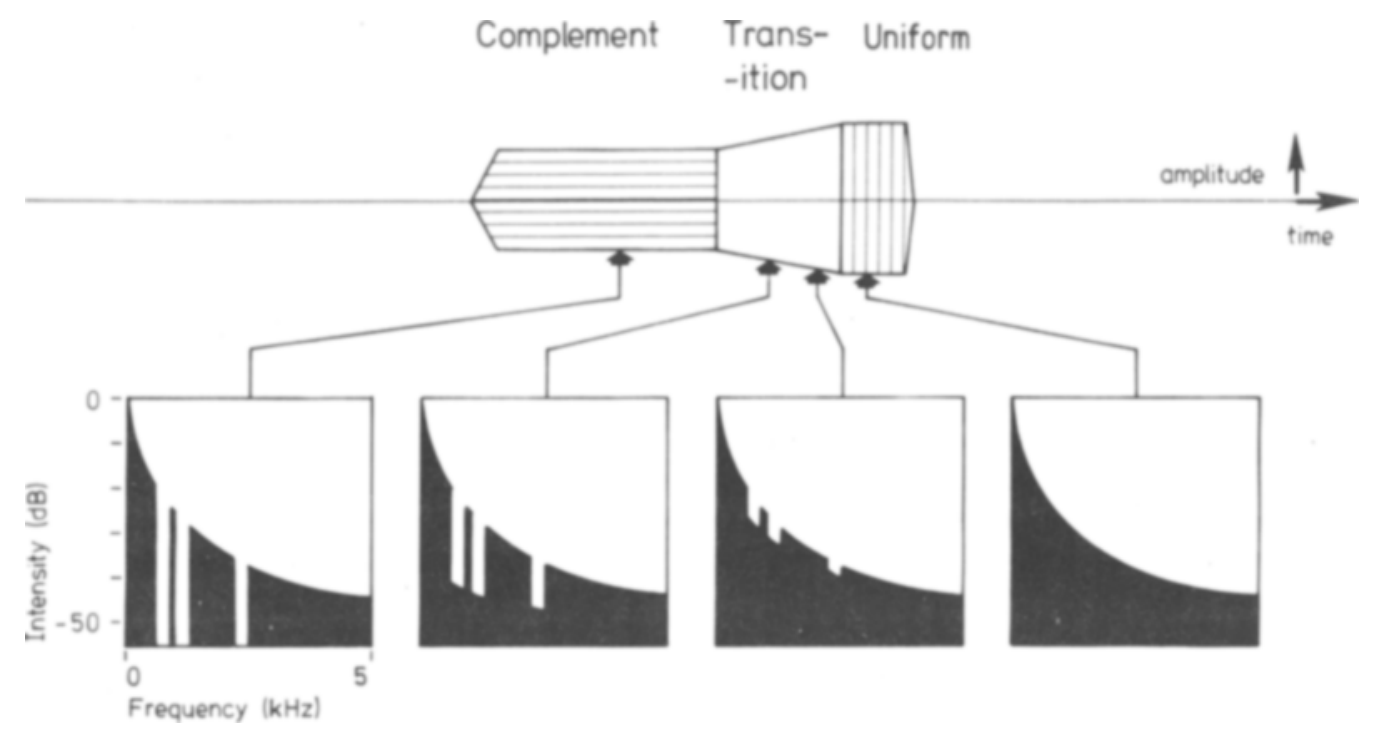

Eigure 3. Schematic representation of a complement-to-uniform (CU) stimulus from Experiment 1. Three segments are demarcated in the waveform envelope. The valleys in the spectrum of the vowel complement are pro. gressively filled during the spectral amplitude transition, leading into the uniform spectrum. To make the nature of the spectral amplitude transition clear, the durations of the three segments have not been drawn to scale.

rise time was $100 \mathrm{msec}$, and the fall time was $25 \mathrm{msec}$. One test stimulus of this type was created for each vowel complement. Two foils were created from each test stimulus by following the vowel complement with a spectral amplitude transition leading into an indistinct vowel in the place of the uniform spectrum. In the uniform-to-complement (UC) stimuli, the order of the segments was reversed. Of the uniform spectrum, $275 \mathrm{msec}$ was followed by a 25-msec spectral amplitude transition leading into $1,000 \mathrm{msec}$ of a vowel complement. The rise time was $25 \mathrm{msec}$, and the fall time was $100 \mathrm{msec}$. Ten foils were created by replacing the uniform spectra with indistinct vowels.

Separate randomizations were recorded for the $\mathrm{CU}$ and $\mathrm{UC}$ stimuli. In each randomization, the five test stimuli each occurred 10 times and the 10 foils each occurred five times. Trials occurred at 5-sec intervals. Three randomizations of control stimuli were also recorded. The first contained 10 instances of each of the five 300 -msec vowels used in the screening test. The second contained 10 instances of analogous 300 -msec stimuli derived from the indistinct vowel segments. The third contained 10 instances of 300 msec stimuli derived from the vowel complements and 10 instances of a 300-msec segment of the uniform spectrum.

The subjects listened first to the tape containing the vowel stimuli, second to the indistinct vowels, and third to the complements and uniform spectrum. They were instructed to identify each of the stimuli presented as one of the five sounds, "ee," "ah," "oo," "or," and "er." The subjects then listened to the CU stim- uli. They were instructed to identify the onset of the second segment (the uniform spectrum) in one of the same five categories. Finally, they listened to the UC stimuli and were instructed to identify the offset of the first segment in one of the five categories ignoring the quality of its onset.

\section{Results and Discussion}

Results, averaged over the six subjects, are given in Table 2. The first row shows that these listeners were by now $100 \%$ accurate when identifying the five vowel stimuli. Four of the six also identified the indistinct vowels (row 2) without error, and one subject was $\mathbf{9 0 \%}$ correct and the other $\mathbf{8 8 \%}$ correct. Overall, these scores were high enough to establish that the indistinct vowels would be effective as foils. Row 3 lists the percentages of occasions on which the segment with the uniform spectrum was identified as the vowel whose complement preceded it in the $\mathrm{CU}$ condition. Performance was above chance for each of the vowels individually. The foils were identified accurately $(94 \%)$, suggesting that subjects followed the instructions and attempted to identify the onset

Table 2.

Results of Experiment 1 : Percentages of Correct Identification Responses in Each Vowel Category for Four Types of Stimulus [Vowels, Indistinct Vowels, Complement-Uniform (CU), Uniform-Complement (UC)] and the Absolute Percentage of Responses in Each Category Made When the Uniform Spectrum was Presented in Isolation, Averaged Over Six Subjects

\begin{tabular}{|c|c|c|c|c|c|c|c|}
\hline \multirow[b]{2}{*}{ Stimulus } & \multicolumn{5}{|c|}{ Response Category } & \multirow[b]{2}{*}{ Mean } & \multirow[b]{2}{*}{ Foils } \\
\hline & EE & $\mathbf{A H}$ & oo & OR & ER & & \\
\hline Vowels & 100.0 & 100.0 & 100.0 & 100.0 & 100.0 & 100.0 & \\
\hline Indistinct Vowels & 95.0 & 98.3 & 95.0 & 95.0 & 98.3 & 96.3 & \\
\hline $\mathrm{CU}$ & 98.3 & 96.7 & 83.3 & 71.7 & 78.3 & 85.7 & 94.3 \\
\hline UC & 0.0 & 48.3 & 33.3 & 28.3 & 15.0 & 25.0 & 94.0 \\
\hline Uniform & 1.7 & 31.7 & 10.0 & 45.0 & 11.7 & , & \\
\hline
\end{tabular}


of the second segment, not basing their responses on conscious inference from the perceived identity of the complements. In the UC condition, in row 4, performance was poor, although the foils were again identified accurately. It was not possible to identify the offset of the uniform spectrum as the vowel whose complement followed it. (The two subjects who were also authors listened several times to this tape but could not perceive the predicted vowel sound from the spectral amplitude transition leading into the vowel complement.) The fifth row of Table 2 lists as a control the percentages of responses in each of the five vowel categories made when the uniform spectrum was presented in isolation. Comparison of rows 4 and 5 suggests that listeners identified the uniform spectrum in Condition UC in essentially the same way as they identified it in isolation.

Experiment 1 demonstrates two things. First, the basic phenomenon, in which a segment with a uniform spectrum is heard as the vowel whose complement abuts it, has been shown to generate a range of predictable vowel percepts. However, vowels are heard only when the complement precedes the uniform spectrum. The result offers no support for the most general formulation of the spectral change hypothesis, although it remains possible that only positive-going changes (i.e., those at the onset of the uniform spectrum) are perceptually relevant. The perceptual grouping hypothesis could be formulated to encompass the obtained time asymmetry, but at the expense of parsimony, and the adaptation hypothesis predicts the result obtained.

In the introduction, we noted that the three hypotheses are not directly opposed. To explore the relevance of each in their respective domains, we adopted an indirect approach. Experiment 2 explores the consequences of manipulating three parameters: the duration of the vowel complement, the separation of the complement and the uniform spectrum, and, in order to measure the extent of interaural transfer of the effect, the ear to which complement and uniform spectrum were presented. The time asymmetry of the present results using vowel identification and the general nature of the effects obtained by Wilson (1970) and Viemeister (1980) with nonspeech stimuli favor an account in terms of adaptation. However, a relatively natural task, such as vowel identification, could engage other, higher level processes in addition to lower level effects of adaptation. If so, the present effects may either emerge in situations where the corresponding psychoacoustic phenomena with nonspeech stimuli have not been found or may display aspects not explicable by adaptation.

\section{EXPERIMENT 2a}

Wilson (1970) found that the aftereffect produced by a comb-filtered noise is reduced by about $80 \%$ after 0.5 to $1 \mathrm{sec}$. The pattern of decay with time was approximately linear in log time and could therefore be extrapolated to determine the putative duration at which it would have decayed completely. This duration varied with the duration of the preceding combfiltered noise and was about $0.75 \mathrm{sec}$ following a 0.25 -sec exposure, $1.25 \mathrm{sec}$ following a $1.0-\mathrm{sec}$ exposure, and $3.0 \mathrm{sec}$ following a 4.0-sec exposure. Viemeister (1980) measured a more gradual rate of decay for the adaptation of masking produced by an incomplete harmonic complex. About $20 \%$ of the effect remained $6.4 \mathrm{sec}$ after exposure to a 2.4-sec adaptor.

In Experiment $2 a$, the duration of the interval between the end of a 1.0-sec vowel complement and the onset of the uniform spectrum was varied. In one condition, this interval was silent; in the second condition, it was occupied by a spectral amplitude transition. We reasoned that, if a version of the spectral change hypothesis held, there might be an optimal rate of spectral amplitude change for producing percepts of vowels-other than the notionally infinite rate achieved when vowel complements directly abutted the uniform spectrum. There are general grounds for expecting such optima in rate-sensitive systems (e.8., Moller, 1972). Maximum accuracy in vowel identification might then occur at some greater-than-zero separation of vowel complement and uniform spectrum. If not, or if the optimum is the fastest rate of change achievable, then greatest accuracy should occur when vowel complements directly abut the uniform spectrum.

\section{Method}

Two types of stimuli were derived from the complement-touniform stimuli in Experiment 1. In each, $1,000 \mathrm{msec}$ of a vowel complement with a 100 -msec rise time preceded 275 msec of the uniform spectrum with a 25 -msec fall time. In the variabletransition stimuli, a linear spectral amplitude transition linked the vowel complement to the segment with the uniform spectrum. The duration of the transition was $1,8,16,32,64,128,256$, or $512 \mathrm{msec}$. In the variable-silence stimuli, the spectral amplitude transition was replaced by silence. The fall time of the vowel complement and the rise time of the uniform spectrum were $1 \mathrm{msec}$. Complements of only three of the former set of vowels were used: $/ i /, / a /$, and $/ u /$. Two sets of foils in which indistinct vowels replaced segments with uniform spectra were also synthesized. Separate randomizations were recorded for the variable transition and variable silence stimuli. Ten occurrences of each of the 24 test stimuli and five occurrences of each of the 48 foils occurred in each randomization. The six subjects were instructed to identify the onset of the second segment in each stimulus as one of the sounds "ee," "ah," and "oo."

\section{Results and Discussion}

Results of the variable-transition condition are displayed in the upper panel of Figure 4. Results of the variable-silence condition are shown in the lower panel. In each panel, the percentage of occasions on which the uniform spectrum was identified as the vowel whose complement preceded it has been plotted against the duration of silence or transition. Data 
have been plotted for each complement individually. In each condition, the foils were identified accurately (96\% in the variable-transition condition and $100 \%$ in the variable-silence condition).

There are four relevant aspects of the results. First, in general, performance was best when vowel complements and uniform spectra abutted one another with no separation, and performance declined as the separation was increased. Second, performance appears to have been consistently good with the complement of the vowel $/ a /$. This probably occurred because of an overall bias due to the uniform spectrum itself sounding like "ah" (or "or"), as the results of Experiment 1 showed. Results obtained with the other two complements will be more informative of performance, therefore. In the variable-silence condition, accuracy of identification of the two other
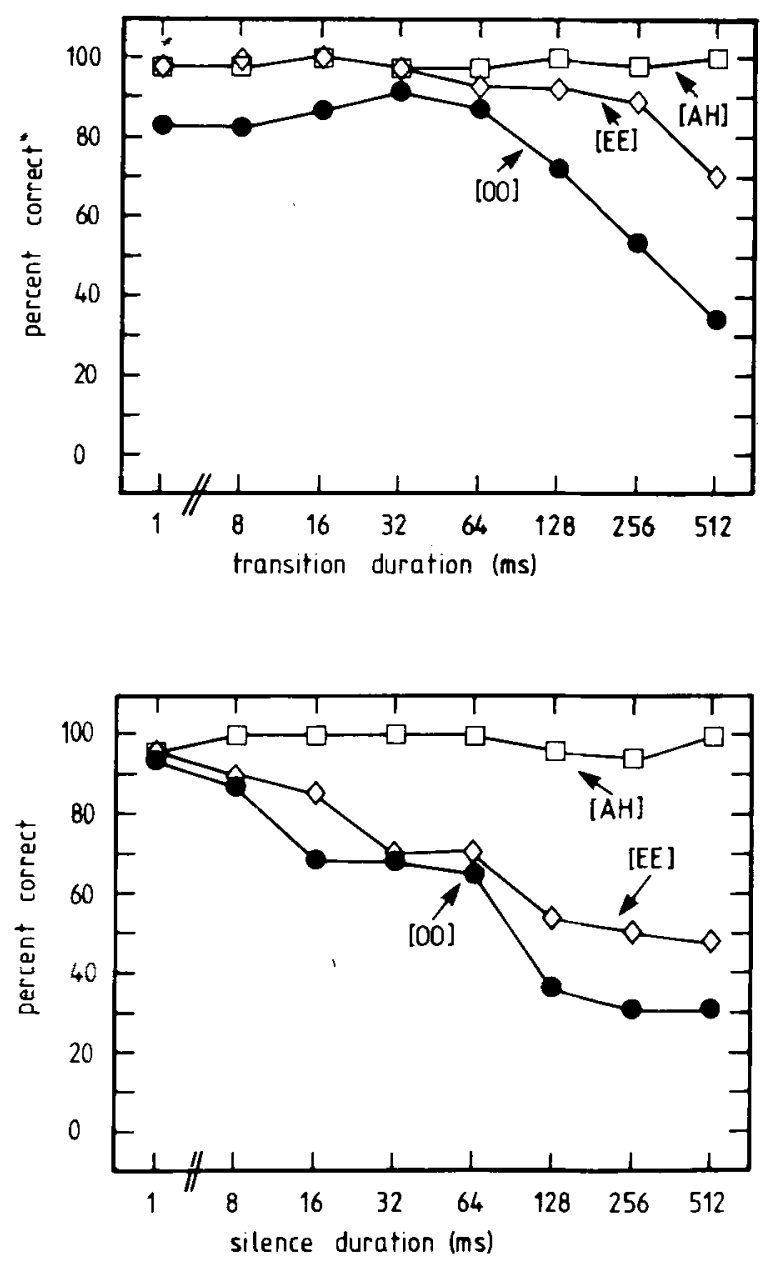

Figure 4. Results of Experiment 2a averaged over six subjects. The percentage of occasions on which a segment with a uniform spectrum was "correctly" Identified as the vowel whose complement preceded it is plotted aguinat the duntion of the spectral amplitude transition (upper panel) or the duration of the silent interval (lower panel) which linked the vowel complement and the uniform spectrum. Results for three vowel complements are displayed separately. Each point plots the results of 60 observations. vowels, $/ \mathrm{i} / \mathrm{and} / \mathrm{u} /$, fell to $50 \%$ with approximately $100 \mathrm{msec}$ of silence, ranging across subjects from 20 to $512 \mathrm{msec}$. Third, there was no evidence of an optimal duration for a linear spectral amplitude transition. The maximum apparent in the mean data for $/ \mathrm{u} /$ with $32 \mathrm{msec}$ of transition was evidenced by only two individual subjects. Finally, performance at the longer separations was better in the variable transition condition.

Overall, the results obtained in the variable-silence condition suggest that the effect of a vowel complement decays within, at most, $500 \mathrm{msec}$. This is a shorter decay time than the analogous durations measured by Wilson (1970) and Viemeister (1980). There is no evidence here of cognitive processes of selective attention or judgmental contrast sustaining the effect beyond the duration expected from the adaptation component alone. It seems likely, therefore, that performance was better in the variabletransition condition because some portion of the spectral amplitude transition acted as a continuation of the complement. In other words, although the intensity contrast would be reduced, it would be shifted nearer to the onset of the segment with the uniform spectrum. Below, in Experiment 3, this possibility is explored by determining the minimal amount of spectral amplitude structure required in a vowel complement to elicit the effect.

\section{EXPERIMENT 2b}

\section{Method}

Viemeister (1980) determined that the adaptation of masking produced by an incomplete harmonic series develops with 50 to

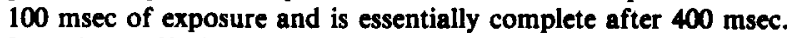
Experiment $2 \mathrm{~b}$ determined the duration of vowel complement required to produce the present effect. Ten complement durations were used, ranging from 25 to $250 \mathrm{msec}$ in 25 -msec steps. The rise time of the complement was $25 \mathrm{msec}$. The duration of the spectral amplitude transition was $1 \mathrm{msec}$ and the duration of the segment with the uniform spectrum was $275 \mathrm{msec}$ with a 25 -msec fall time. Complements of the three vowels $/ i /, / a /$, and $/ u /$ were used. Two foils were constructed with each complement. A randomization was recorded in which each stimulus occurred eight times. The six subjects were instructed to identify the onset of the second segment as one of the three vowel sounds "ce," "ah," and "oo."

\section{Results and Discussion}

In Figure 5, the percentage of times that a segment with a uniform spectrum was identified as the vowel whose complement preceded it has been plotted against the duration of the complement. Data for each vowel complement are presented averaged over the six subjects. The accuracy with which the foils were identified was $97 \%$. Performance with the complement of $/ u /$ at long durations has been depressed by the performance of two of the subjects, who consistently failed to identify the following uniform spectrum as "oo." The other four subjects produced consistent data and identified "ee" and "oo" above $50 \%$ correct with between 75 and $150 \mathrm{msec}$ of 


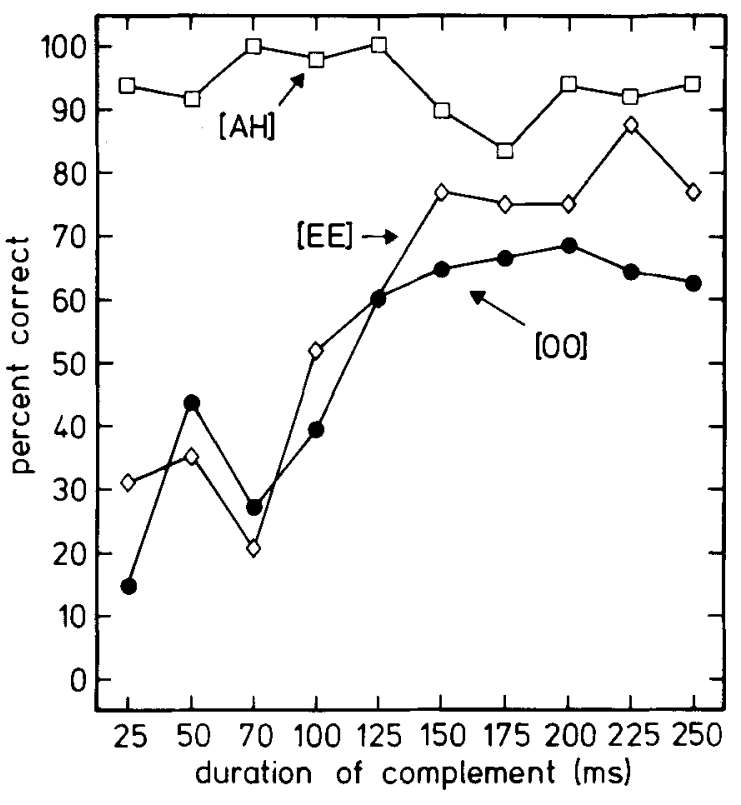

Figure 5. Results of Experiment $2 b$ averaged over six subjects. The percentage of occasions on which a segment with a uniform spectrum was "correctly" Identified as the vowel whose complement preceded it is plotted against the duration of the complement. Results for three vowel complements are displayed separately. Each point plots the results of 48 observations.

complement. Acknowledging the different procedures and criteria of correct performance, this range is similar to the range of 50 to $100 \mathrm{msec}$ established by Viemeister (1980) as being necessary to produce adaptation of masking. As in Experiment 2a, there is no evidence that the speech-identification task has allowed cognitive processes to extend the effect beyond the temporal limits expected from its adaptation component.

\section{EXPERIMENT 2c}

Viemeister and Bacon (1981) suggested that their aftereffect does not result from central processes because it does not transfer between the ears, although they presented no formal data on this point. In Experiment 2c, $995 \mathrm{msec}$ of the complement of one of the five vowels $/ \mathrm{i} /, / \mathrm{a} /, / \mathrm{u} /, / \mathrm{s} /$, and $/ 3 /$ preceded $295 \mathrm{msec}$ of the uniform spectrum. The complement could occur in either the left or the right ear; the uniform spectrum could occur in either the ipsilateral or the contralateral ear. When both were presented to the same ear, they were linked by a 5-msec spectral amplitude transition. When they were presented to opposite ears, the fall time of the complement and the rise time of the uniform spectrum overlapped and were each $5 \mathrm{msec}$. In all conditions, the rise time of the complement was $100 \mathrm{msec}$ and the fall time of the uniform spectrum was $25 \mathrm{msec}$.

We were concerned that the subjects, who were becoming well practiced by now, might realize that even the indistinct vowels provided a set of clearer instances of the vowels than did the uniform spectra following vowel complements. They might, therefore, distinguish the foils from the test stimuli and thereby defeat the object of including the foils. Accordingly, a new set of foils was constructed in which the vowel percept was slightly more ambiguous and less easily distinguished from the percepts produced by the test stimuli. In this new set of vowels, the levels of the three harmonics centered on the frequencies of each vowel formant were reduced by $5 \mathrm{~dB}$ from their levels in the original indistinct vowels.

A randomization was recorded in which each stimulus and each foil occurred five times. The six subjects were instructed to identify the second segment in each presentation as one of the five vowel sounds "ah," s"ee," "oo," "or," and "er."

\section{Results and Discussion}

Table 3 contains the percentages of times that a uniform spectrum was identified as the vowel whose complement preceded it. Results have been collapsed over the five complements, but are reported for each subject individually. Overall, performance was close to $90 \%$ in the monotic conditions and at chance in the dichotic conditions. There is no evidence of any interaural transfer. The foils were identified slightly more accurately when presented dichotically than when presented monotically; this suggests that the poor performance with the dichotic test stimuli did not result from switching attention between the ears or other task factors. It is more likely that the aftereffect of the vowel complements has depressed performance with the foils in the monotic conditions.

The absence of interaural transfer of the present effect is consistent with Viemeister and Bacon's (1981) informal report. Although we acknowledge

Table 3

Results of Experiment $2 c$ for Six Individual Subjects: Percentages of Occasions on Which (1) a Segment With a Uniform Spectrum was Identified as the Vowel Whose Complement had Preceded it (Uniform Spectra) and (2) an Indistinct Vowel Following a

Vowel Complement was Identified Correctly (Foils), for Ipsilateral and Contralateral Presentations of Complements and Test Segments

Test Segment

$$
\text { Uniform Spectra Foils }
$$

Subject Ipsilateral Contralateral Ipsilateral Contralateral

\begin{tabular}{lrrrr} 
Q.S. & 92 & 22 & 62 & 88 \\
J.F. & 98 & 20 & 92 & 98 \\
M.R. & 94 & 22 & 74 & 94 \\
M.F. & 70 & 20 & 56 & 70 \\
P.B. & 100 & 16 & 64 & 88 \\
S.R. & 84 & 18 & 68 & 84 \\
Mean & 89.7 & 19.7 & 69.3 & 87.0 \\
\hline
\end{tabular}

Note-Data have been averaged over five vowel complements and ears. 
that the lack of interaural transfer does not positively confirm any particular peripheral site, it is more parsimonious to conclude that the present effect results from adaptation than to attempt to rescue the perceptual grouping hypothesis by presuming that the underlying mechanisms are monaural.

\section{EXPERIMENT 3}

In Experiment 2a, uniform spectra were identified more reliably when connected to vowel complements by spectral amplitude transitions than when separated by silence. Presumably, the spectral amplitude transition extended the effective duration, and hence extended the proximity to the uniform spectrum, of a complementary adaptor. That conclusion prompts the question of how much spectral amplitude structure is required in a vowel complement to produce an effect, or perhaps more precisely how much temporal amplitude contrast with the uniform spectrum is required around the nominal formant frequencies.

\section{Method}

For Experiment 3a, five new versions of the complement of each of the vowels $/ \mathrm{i} /, / \mathrm{a} /$, and $/ \mathrm{u} /$, in which the depth of the spectral valleys was progressively reduced, were defined. To do this, the log power of each of the nine harmonics in the vowel spectra was reduced by $75 \%, 80 \%, 85 \%, 90 \%$, and $95 \%$ from the values listed in Table 1. The five new complements were defined by subtracting these new levels from the levels of the corresponding harmonics in the uniform spectrum. We shall describe the new vowel complements as entailing $5 \%, 10 \%, 15 \%, 20 \%$, or $25 \%$ of the spectral amplitude structure of the original vowel complements. Test stimuli were synthesized in which $1,000 \mathrm{msec}$ of one of the 15 new vowel complements was followed by a $25-\mathrm{msec}$ spectral amplitude transition leading into a uniform spectrum. Because there was now considerable variation in the sound of the complements, it was not necessary to synthesize foils. A randomization of 150 items was recorded in which each of the 15 test stimuli occurred 10 times.

For Experiment 3b, an analogous set of vowel complements was defined in which the depth of spectral amplitude structure ranged from $1 \%$ to $9 \%$ in $1 \%$ steps. Twenty-seven test stimuli were synthesized, and a randomization of 270 items was recorded, in which each of the test stimuli occurred 10 times. Thus, in the two experiments, spectral structure contrasts ranging from about $1 \mathrm{~dB}$ up to $20 \mathrm{~dB}$ were examined.

The six subjects listened to the tape recorded for Experiment 3a and then to the tape for Experiment $3 \mathrm{~b}$. In each case, they were instructed to identify the onset of the second segment as one of the sounds "ee," "ah," and "oo."

\section{Results and Discussion}

The results of Experiment 3a are displayed in the left-hand panel of Figure 6. The percentage of occasions on which the uniform spectrum was identified as the vowel whose complement preceded it has been plotted against the amount of spectral amplitude structure in the complement for each of the individual complements. Three of the six subjects performed above chance, even on the restricted task of identifying "oo" and "ee," with all of the modulation depths examined. The performance of the other three fell to chance only with the smallest amount of $5 \%$. The right-hand panel of Figure 6 presents the results of Experiment $3 \mathrm{~b}$. Chance performance on the restricted task of identifying "ee" and "oo" occurred at $1.0 \%, 2.1 \%, 4.2 \%, 6.3 \%, 4.4 \%$, and $8.0 \%$, for the six individual listeners. The average is $4.3 \%$. The average difference in the level of the nine formantrelated harmonics between a complement with $4.3 \%$ spectral structure and the uniform spectrum would be $1.6 \mathrm{~dB}$, ranging from $2.2 \mathrm{~dB}(300 \mathrm{~Hz})$ to $1.1 \mathrm{~dB}$ $(2100 \mathrm{~Hz})$.

The minimal spectral structure required to generate the effect is perhaps the most interesting of the present results. Possibly, different degrees of adaptation are produced by spectral components differing in amplitude by $2 \mathrm{~dB}$ around a mean level of $50 \mathrm{~dB}$. Alternatively, if we accept Viemeister and Bacon's (1981) suggestion that the basis of the class of auditory aftereffect examined here is the adaptation of suppression, then suppression must enhance such spectral amplitude variations sufficiently to mean that when suppression adapts and the uniform spectrum is presented, appreciable peaks in the internal auditory spectrum result.

\section{GENERAL DISCUSSION}

The three groups of experiments described here demonstrate that if a segment of a waveform whose spectrum is complementary to that of a vowel (a spectrum with peaks in place of valleys and vice versa) precedes a segment with a uniform spectrum devoid of peaks and valleys, then the uniform spectrum, particularly at its onset, will sound like the vowel whose complement has preceded it. The effect does not occur if the complement follows the uniform spectrum. Approximately $125 \mathrm{msec}$ of the complement of a vowel must precede a uniform spectrum for the effect to emerge, and the effect is eliminated if more than about $500 \mathrm{msec}$ of silence intervene between the complement and the uniform spectrum, or if the complement is presented to one ear and the uniform spectrum to the other ear.

\section{Likely Bases for the Effect}

In terms of the parameters so far explored, the present effect is similar to two effects studied previously using nonspeech sounds. One is Wilson's (1970) demonstration that a broad-band noise presented after exposure to a sinusoidally comb-filtered noise is perceived as possessing a complementary spectral ripple. The other is the demonstration that if a sinusoidal component missing from a harmonic series is reintroduced, then that component is heard clearly against the background of the preexisting harmonics (e.g., Viemeister \& Bacon, 1981). Superficially, it appears that each of these results can be explained by suggesting that the auditory response 


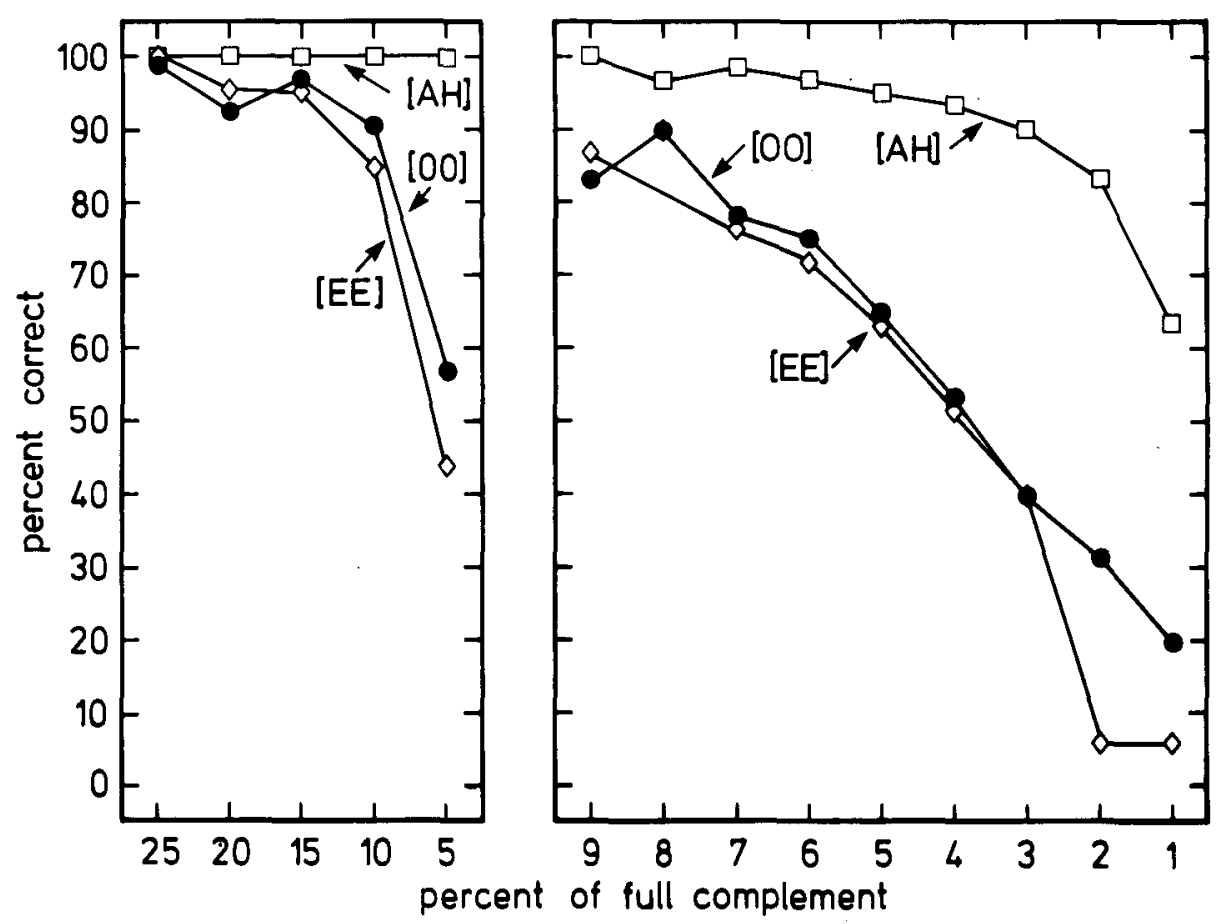

Figure 6. Results of Experiments 3a and 3b averaged over six subjects. The percentage of occasions on which a segment with a uniform spectrum was judged "correctly" as the vowel whose complement preceded it is plotted against the amount of spectral structure in the complement. Results for complements derived from three vowels are displayed separately. Each point plots the results of 60 observations.

diminishes through adaptation at frequencies where there is appreciable energy during the presentation of the adaptor, be it a vowel complement, a combfiltered noise, or an incomplete harmonic series. When the subsequent segment-a uniform spectrum, a broad-band noise, or a complete harmonic seriesis presented, energy at preexisting frequencies achieves a diminished auditory representation relative to energy at new frequencies. The latter stands out, therefore, at least at its onset.

Comparison of the present results with temporal parameters of neural adaptation supports this explanation. At moderate-to-high stimulus levels, the physiological response to the onset of a tone, as reflected in the rate of discharge in the majority of auditory nerve fibers in guinea pig, shows an initially high rate of up to 800 spikes/sec for $1-2 \mathrm{msec}$, which falls rapidly during the next $5-10 \mathrm{msec}$ and asymptotes at a rate between 150 and 200 spikes/sec (e.g., Yates \& Robertson, 1980), but the recovery following the cessation of stimulation is swift. Recovery is complete $150 \mathrm{msec}$ after the cessation of a tone burst. Comparably, about $100 \mathrm{msec}$ of silence eliminated the present effect for four of the six listeners in Experiment 2a.

However, although perhaps a necessary part of an explanation, simple adaptation may not provide a sufficient explanation for the phenomenon. For two listeners, the aftereffect was sustained for up to
$500 \mathrm{msec}$ in Experiment 2a, and analogous effects have been found to last for rather longer, between 3 and $6 \mathrm{sec}$, by Wilson (1970) and Viemeister (1980). This difference in time course suggests that factors other than simple adaptation may play a role.

A possible factor was suggested by Viemeister and Bacon (1981). They demonstrated that the amount of forward masking produced by a reintroduced harmonic is greater than the amount produced by the corresponding member of a complete harmonic series. This finding suggests that the effect does not occur because of a reduction in the auditory response to components present in the adapting sound, but rather that the response to the reintroduced component is enhanced. As an explanation, Viemeister and Bacon suggested that suppression might adapt during the presentation of an incomplete harmonic series. That is, the effectiveness of processes that tend to enhance differences in level in adjacent spectral regions may diminish with exposure to a constant stimulus. As a result, new components are not suppressed relative to their preexisting neighbors, although they will act as suppressors themselves, and so achieve a more potent auditory representation than do old components and a more potent representation than they would achieve if suppression had not adapted. While no aspect of the present results is incompatible with this hypothesis, they should not be construed as providing a direct test of it. At any rate, 
they do tend to confirm that an explanation in terms of peripheral processes is required. Use of a speechidentification task might have been supposed to engage processes underlying perceptual grouping and selective attention that could enhance peripheral effects of adaptation. But the similarity of the time course of effects measured here in Experiment 2 to the analogous effects obtained with nonspeech stimuli by Wilson and by Viemeister, and the absence of an effect in the contralateral ear in Experiment 3, suggests that central effects, if present at all, play a minor role.

One aspect of the present results is difficult to compare with psychoacoustical results because corresponding conditions have not been explored. Experiment 3 demonstrated that, on average, listeners can identify uniform spectra as the vowels whose complements have preceded them with average increase in the levels of the nine harmonics as small as $2 \mathrm{~dB}$. This, result and Viemeister and Bacon's explanatory hypothesis deserve further study.

\section{Potential Roles for the Present Effect}

Processes of rapid adaptation tend to enhance onsets selectively, while processes of suppression are usually regarded as preserving and possibly enhancing differences in level in adjacent spectral regions in the auditory representation of complex spectra (e.g., Houtgast, 1974; Moore \& Glasberg, 1983). The present results emphasize two other potential roles. Either simple adaptation or the adaptation of suppression could serve to enhance changes in spectral amplitude when energy occurs in spectral regions where immediately previously there was less energy. In speech, therefore, changes in the spectral distribution of energy would be generally enhanced. This process could play a specific role in the perception of syllable-final formant transitions. At the beginning of the transitions, changes in frequency will make formants contrast with what were valleys in the preceding vowel. At the end of the transitions, overall intensity will tend to be low and the absence of any offset effect in Experiment 1 means that their discriminability will not be enhanced by the mechanism enhancing spectral representation of onsets. Any contrastive enhancement of the former type will therefore be valuable.

More generally, from the ecological point of view, the phenomenon observed here may reduce the deleterious consequences of poor acoustical environments and low-fidelity communication channels. Being selective for change, preprocessing of the type achieved by simple adaptation, or the adaptation of suppression, tends to reduce the effect of static or statistical long-term spectral peaks and valleys upon the internal auditory representation, while enhancing the effect of the time-varying information that is of communicative or other biological significance. Responsiveness to change may also assist the short-term process of extracting speech signals from background noises. Where a noise has a relatively static RMS amplitude, the envelope variation due to the speech at the informative low modulation frequencies will be greater than that due to the noise. The changesensitive mechanism will then enhance the effective signal-to-noise ratio, especially since, in speech, the informative modulation patterns in different critical bands are correlated. (See Hall et al., in press.) The ease with which we have tapped into this psychoacoustic phenomenon using a linguistic response suggests that central auditory analysis takes specific advantage of this apsect of peripheral preprocessing.

\section{REFERENCES}

Dannenbring, G. L., \& Bregman, A. S. (1978). Steaming vs. fusion of sinusoidal components of complex tones. Perception \& Psychophysics, 24, 369-376.

Darwin, C. J. (1983). Auditory processing and speech perception. In H. Bouma \& D. G. Bouwhuis (Eds.), Attention and performance $X$. Hillsdale, N.J: Erlbaum.

Hall, J. W., Haganed, M. P., \& Fernandes, M. A. (in press). Detection in noise by spectro-temporal pattern analysis. Journal of the Acoustical Society of America.

HouTG A8т, T. (1974), Lateral suppression in hearing: A psychophysical study of the ear's capability to preserve and enhance spectral contrasts. Soesterberg, The Netherlands: Institute for Perception TNO.

KoFrka, K. (1922). Perception: An introduction to Gestalt Theorie. Psychological Bulletin, 19, 551-585.

Marr, D. (1982). Vision. San Francisco, CA: Freeman.

Molle R, A. (1972). Coding of amplitude and frequency modulated sounds in the cochlear nucleus of the rat. Acto Physiologica Scandinavica, 86, 223-238.

Moone, B. C. J., \& Glasberg, B. R. (1983). Masking patterns for synthetic vowels in simultaneous and forward masking. Journal of the Acoustical Society of America, 73, 906-917.

Viemeisten, N. F. (1980). Adaptation of masking. In G. van den Brink \& F. A. Bilsen (Eds.), Psychophysical, physiological, and behavioural studies in hearing (pp. 190-198). Delft: Delft University Press.

VIEMEISTER, N. F., \& BACON, S. P. (1981). Forward masking by enhanced components in harmonic complexes. Journal of the Acoustical Society of America, 71, 1502-1507.

WILson, J. P. (1970). An auditory after image. In R. Plomp \& G. F. Smoorenburg (Eds.), Frequency analysis and periodicity detection in hearing, (pp. 303-315). Leiden : A. W. Sijthoff.

YATES, G. K., \& RoBE RTSON, D. (1980). Very rapid adaptation in auditory ganglion cells. In G. van den Brink \& F. A. Bilsen (Eds.), Psychophysical, physiological, and behavioural studies in hearing. Delft: Delft University Press.

ZwICKER, E. (1964). "Negative afterimage" in hearing. Journal of the Acoustical Society of America, 36, 2413-2415.

(Manuscript received October 17, 1983; revision accepted for publication December 22, 1983.) 